\title{
Diagnostic Implications of Multi-Photon Fluorescent Microscopy
}

\author{
C.L. Phillips ${ }^{1}$ and V.H. Gattone ${ }^{2}$
}

Pathology ${ }^{1}$ and Anatomy \& Cell Biology ${ }^{2}$, Indiana University School of Medicine, Indianapolis, IN 46202, USA

Day-to-day clinical practice of anatomic pathology relies almost exclusively on the use of microscopes. Brightfield or "light" microscopy is the gold standard desk-top visualization technique for pathologists, providing an affordable and reliable method to rapidly screen and diagnose human disease. Widefield fluorescent and transmission electron microscopes require advanced training at the technical and interpretative level and are usually relegated to esoteric subspecialties focused on kidney, skin and neurological disease. Rapidly expanding knowledge from the fields of genomics and proteomics is influencing pathologic diagnosis at unprecedented rates such that the subspecialty of molecular pathology supplements microscopic interpretation. Pathologists with expertise in cellular and molecular biology are poised to embrace new technologies and translate genomics and proteomics into diagnostically useful tests. One such technology is multi-photon fluorescent microscopy, a state of the art imaging system for which pathologists are particularly well-suited. Two-photon fluorescent microscopes, when combined with fluorescent probes and volume rendering software, permit three-dimensional (3-D) exploration of structures embedded within thick biological specimens such as surgical pathology samples. To determine the utility of this technique for tissue diagnosis, we exploited methods our laboratories have previously established for specimens fixed with aldehyde-based solutions [1-3]. As an example, we applied these techniques to visualize normal and dilated renal tubules and ducts in the Balbc/cpk mouse model of cystic kidney disease. Thick vibratome tissue sections were prepared from intact kidneys of 7-day-old mice and dual-labeled with fluorescent probes. Using Bio-Rad and Zeiss twophoton microscopes at the Indiana Center for Biological Microscopy (http://web.medicine.iupui.edu/icbm/), optical Z-series were collected with water-immersion objectives in $\mathrm{X}, \mathrm{Y}$ and $\mathrm{Z}$ axes. Optical Z-series were rendered in 3-D with Voxx software (freeware, http://web.medicine.iupui.edu/icbm/software/). A composite of images (panel A) was assembled in Adobe PhotoShop (San Jose, CA). We demonstrated the 3-D relationship of cystic collecting ducts to neighboring convoluted proximal tubules of normal caliber as well as the nature of the collecting duct dilations in this early stage cystic kidney disease. Cystic kidney disease is a good proof-ofprinciple disease as the pathology is robust and progressive, yet tubule segment-specific. Cystic epithelial cells also undergo some dedifferentiation and, as shown in panels B \& C, it is possible to detect different levels of lectin binding suggestive of changes in the stage of cellular differentiation. Overall cellular detail and tissue architecture were sufficiently preserved in situ such that image volumes could be viewed on a standard resolution computer monitor. With Voxx software the individual volumes could be rotated and visually explored in 3-D. In conclusion, the combination of two-photon microscopy, selective fluorescent markers and volume rendering software provides useful information on overall 3-D tissue architecture that has potential diagnostic utility for unmasking complex lesions embedded within human specimens. Pathology laboratorybased human tissue banks could be mined as a rich source of material appropriate for such 3-D morphological and molecular analysis. Anatomic pathology specimens are no longer limited to analysis with conventional two-dimensional histological stains and brightfield microscopes. 


\section{References}

[1] C.L. Phillips et al., Amer J Pathol 158: 49-55 (2001)

[2] J.L. Clendenon et al., Am J Physiol Cell Physiol 282: C213 (2002)

[3] C.L. Phillips et al., J Am Soc Nephrol. 15(7):1744-55 (2004)
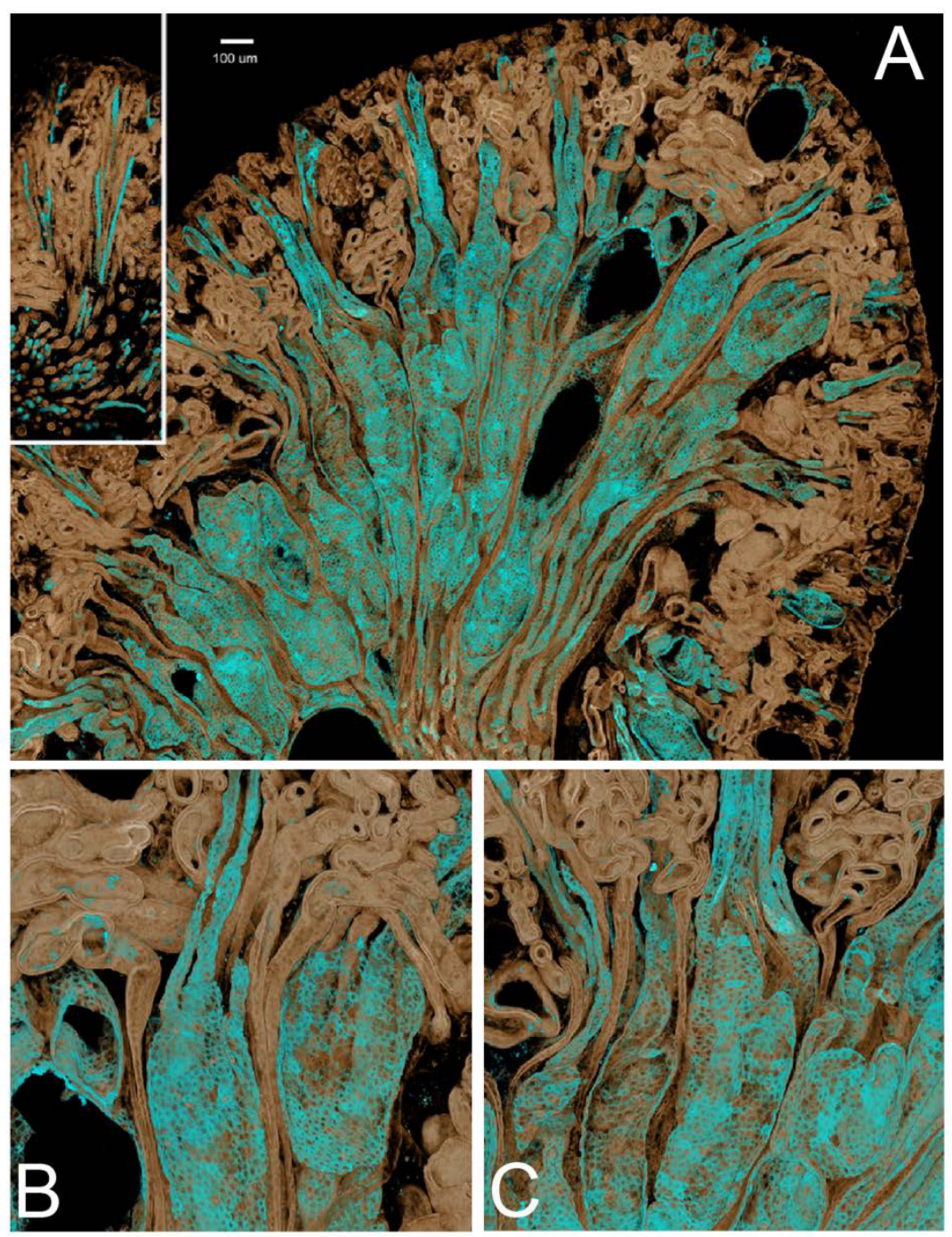

Figure: Two-photon microscopy of mouse kidneys. Vibratome sections were dual-labeled with fluorescently tagged lectins. Cortical proximal tubules were decorated with peanut agglutinin lectin (pseudocolored brown). Collecting ducts were labeled with Dolicho biflorus lectin (pseudocolored blue). Panel A, a composite of 27 volumes, was assembled to show the intricate relationship of proximal tubules to collecting ducts. Cortical proximal tubules in Balbc/cpk mice are of expected orientation and uniform caliber, similar to tubules in the normal control (inset, upper left). Collecting ducts radiate from medulla into the cortex in their expected alignment but have dilated lumina. In panels B and C, the S3 segments of proximal tubules enter the medulla as they lose their convolutions and exhibit a straighter vertical alignment as they transition to thick descending limbs. In panel B, two collecting ducts are seen to unite in the medulla where luminal diameter is more profoundly dilated. 